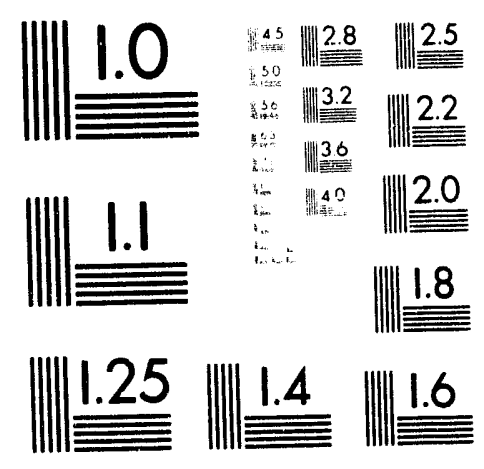



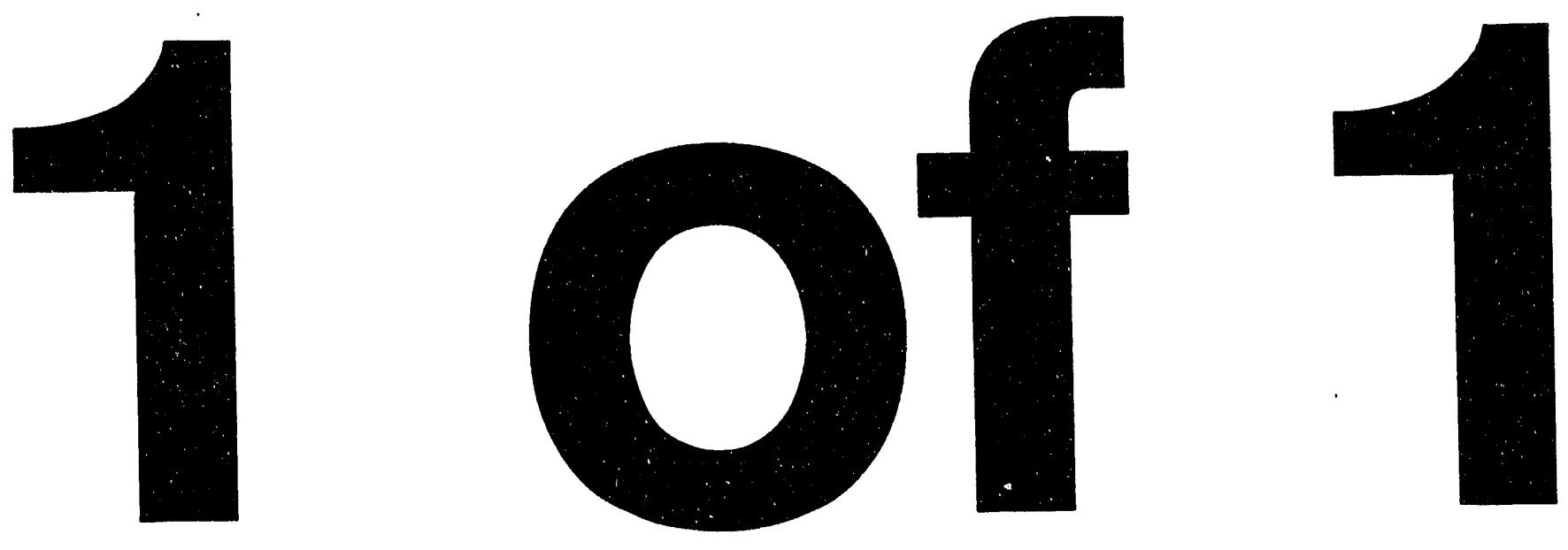
BNL- 49313

CAP 100-ATF-93C

\section{Development of the Alternate Entry Port for the ATF}

Zohreh Parsa

Physics Department, 901A

May 1993

\section{DISCLAIMER}

This report was prepared as an account of work sponsored by an agency of the United States Government. Neither the United States Government nor any agency thereof, nor any of their employees, makes any warranty, express or implied, or assumes any legal liability or responsibility for the accuracy, completeness, or usefulness of any information, apparatus, product, or process disclosed, or represents that its use would not infringe privately owned rights. Reference hercin to any specific commercial product, process, or service by trade name, trademark, manufacturer, or otherwise does not necessarily constitute or imply its endorsement, recommendation, or favoring by the United States Government or any agency thereof. The views and opinions of authors expressed herein do not necessarily state or reflect those of the United States Government or any agency thereof.

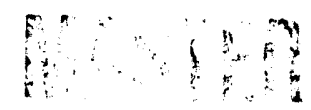




\title{
Development of the Alternate Entry Port for the ATF*
}

\author{
Zohreh Parsa \\ Physics Department \\ Brookhaven Vational Laboratory, \\ Upton, VY 11973
}

Abstract

We discuss a second entry port for the Accelerator Test Facility (ATF) injection system at Brookhaven National Laboratory, which consists of a photocathode if gan and a straight - ahead beamline directly into the 50 $\mathrm{MeV}$ linac. The proposed second entry port should improve the beam quality and lower the emittance needed for FEL (Free Electron Laser), and laser - acceleration experiments. A discussion on the laser driven high brightness photoelectrons through the primary entry port (a low energy 180 degrees achromatic double bend transport line) now in operation, and a beam analysis for the proposed secondary port is also given.

\section{INTRODUCTION}

The high brightness laser driven photoelectron beams (with low emittance and high current) are required for new methods of acceleration such as FEL, IFEL (an inverse free-electron laser) and future development of linear colliders. To achieve high brightness and rapid acceleration, an of gun operating at $2.856 \mathrm{GHz}$, with $1-1 / 2$ cells, $\pi$ - mode resonant, disc-loaded structure (with cathode placed at the start of the first $1 / 2$ cell), has been designed and operating as the injector for the two $S$-band linac sections at the BNL Accelerator Test Facility (ATF). The cathode is illuminated with a frequency quadrupled $\mathrm{Nd}$ :YAG laser with photon energy of $4.65 \mathrm{MeV}$ at the gun exit.

The Accelerator Test Facility at BNL is an experimental Facility for accelerator and beam physics experiLtents. A major research interest is in the interaction of laser driven photoelectrons with the electromagnetic fields. ATF's present injection system consist of a photoelectron gun and a low energy transport beamline which consists of two sets of quadrupole triplets and a $180^{\circ}$ achromatic double bend, (Fig. 1). The photoelectrons are ejected from a photocathode if gun into the transport beam line and then accelerated through $2 \mathrm{~S}$ - band linac sections to $50 \mathrm{MeV}[1,2]$. Thu inac consists of two $\pi / 3$ - mode, $3.05 \mathrm{~m}$ long, disc loaded, traveiling wave SLAC linear accelerator sections that was produced at IHEP, Beijing,

China. The gun and the lir ac are driven by the same XK5 klystron, delivering about $25 \mathrm{MW}$ of peak power with a 3.5 micro-second pulse at $2.856 \mathrm{GHz}$ [2].

With the existing injection system, the beam diverges quckly at the gun exit and gets large in going through the double bend transport line into the linac $[3,4]$. We have considered and studied various schemes $\{6\}$ and will present some results for the proposed ATF aiternate injection

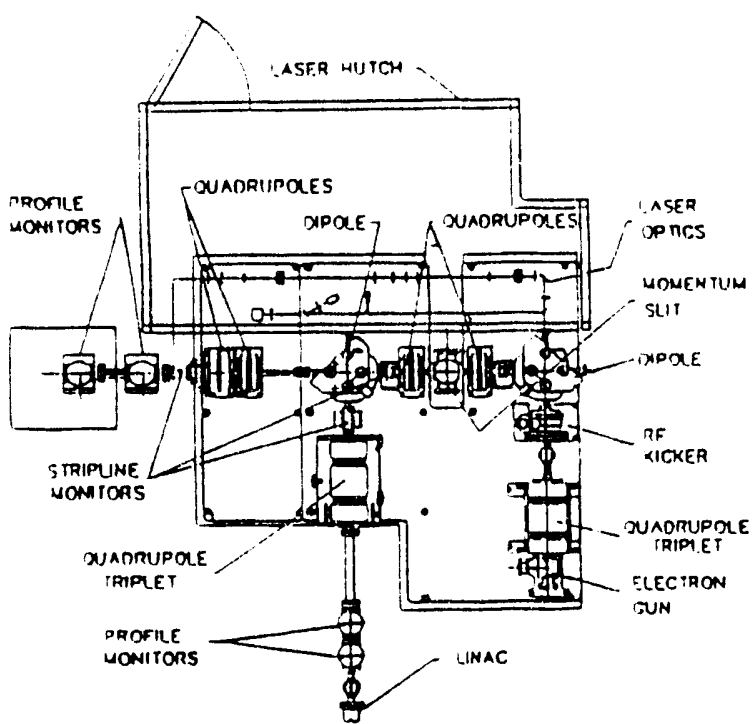

Figure 1: Sketch of the BNL ATF injection system. The transport beamline from the gun to the linac entrance showing the positions of the magnetic elements.

system in section II. A summary and references are given in section III.

\section{ALTERNATE INJECTION SYSTEM}

We have considered various schemes to improve the beam quality and preserve the low emittance and high brightness beam needed for experiments at ATF [6!. In this section we present some of our calculations and simulation results obtained for a 2 nd entry port for ATF. The alternate (straight-ahead) injection system consists of a solenoid + a gun + solenoid combination that is placed directly into the linac.

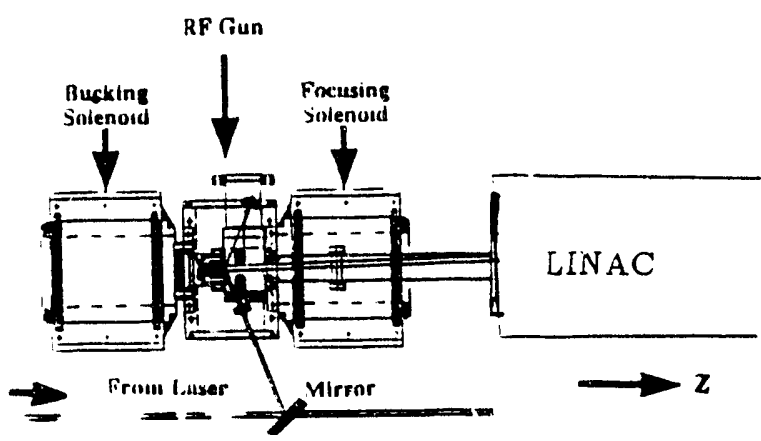

Figure 2: Sketch of the alternate injection system for ATF. A solenoid + gun + solenoid $($ gun $+s)$ combination is placed in line with the linac. (Not scaled). 

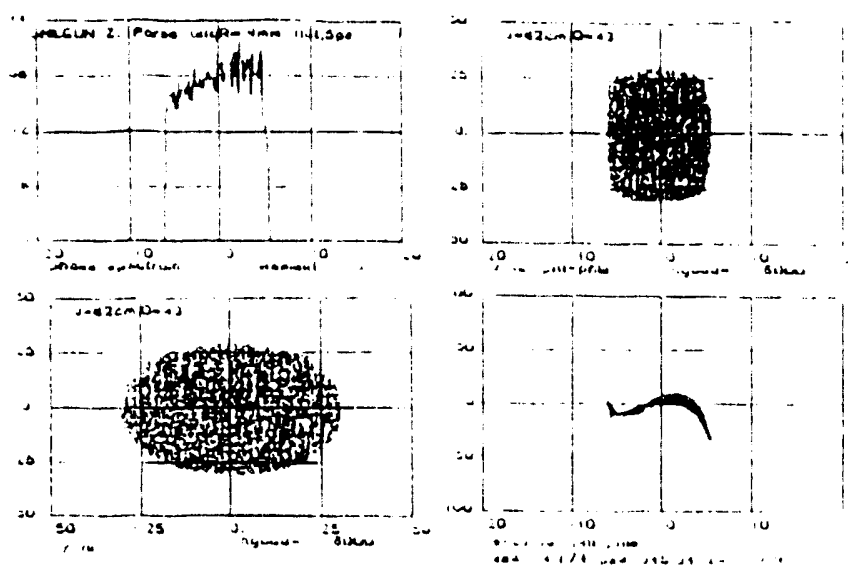

Figure 3a: Shows the beam profile at $z=7.9 \mathrm{~cm}$ (exit of the gun cavity). From the top in c.w.direction: phase spectrum, $x$ vs change in phase, $y$ vs $x$ profile and change in energy vs change in phase with program PARMELA.
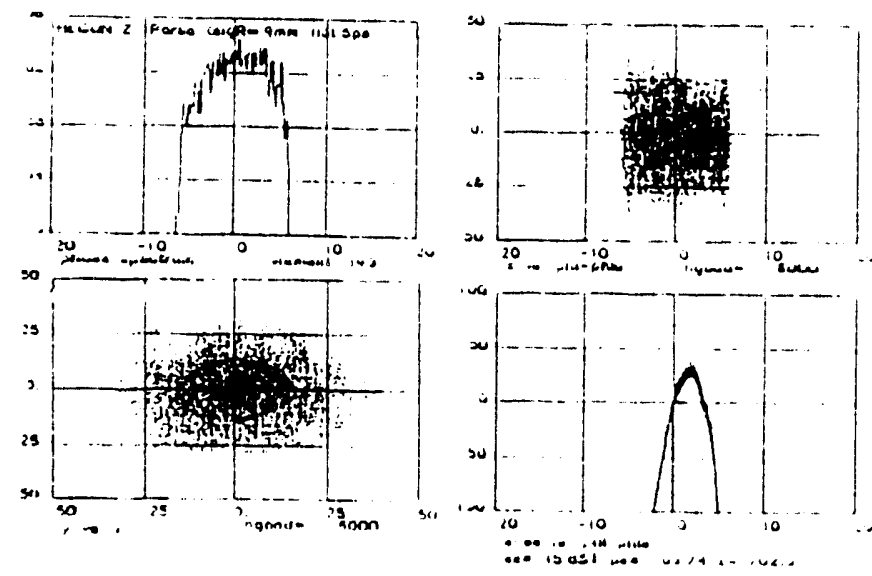

Figure 3b: Shows the beam profile at $z=702.3 \mathrm{~cm}$ (the linac exit). From the top in c.w.direction: phase spectrum, $x$ vs change in phase, $y$ vs $x$ profile and change in energy vs change in phase with program PARMELA.

This scheme would allow the production of the low emittance and high brightness beam needed for the experiments and preserves the present injection system which can be used alternatively for diagnostic measurements etc. Where the beam is injected from a 2 nd entry port (using a 2nd gun) directly into the linac without going through the bending magnets.

Selections of $\mathrm{d}$ the distance from the gun (cathode) to the linac entrance and solenoid strength are important and would effect beam quality (see Section III). For other injection schemes and a more detailed analysis see refereaces. In this analysis the fields off axis for solenoids are calculated to sixth order using the expansions:

$$
\begin{aligned}
B:(z, r) & =B:(z, 0)-\frac{r^{2}}{t} \frac{d^{2} B}{d z^{2}} \\
& -\frac{r^{2}}{4}\left(\frac{d^{4} B}{d z^{4}} \frac{r^{2}}{16}-\frac{d^{6} B}{d z^{6}} \frac{r^{1}}{576}\right)
\end{aligned}
$$

$$
B_{r}(z, r)=-\frac{r}{2}\left(\frac{d B}{d z}-\frac{d^{3} B}{d z^{3}} \frac{r^{2}}{8}-\frac{-d^{5} B}{d z^{3}} \frac{r^{4}}{192}\right)
$$

\section{WI. BEAM DYNAMICS AND ANALYSIS}

In our analysis of the beam from cathode through the linac exit we have considered various initial charge distributions, laser spot sizes $\left(\sigma_{+}\right)$, laser pulse lengths $\left(2 \sigma_{z}\right)$, field on the cathode $E$ and accelerating field of the linac, etc. $[6]$. Table 1 illustrates some of our results obtained with the initial parameters of interest for ATF and the proposed UVFEL project at BNL. For example with initial $E=100 \mathrm{MV} / \mathrm{m}$ on the cathode, laser pulse

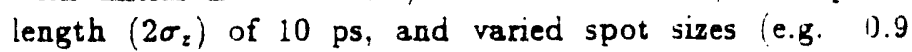
$\mathrm{mm}$ ), and various values of $\mathrm{d}$ (e.g. $62 \mathrm{~cm}$, the distance from cathode to linac entrance), solenoid strength of 2.2 KG and initial phase of 43 degrees we can preserve the beam quality and achieve high brightness, low emittance beam at the linac exit, which is needed for the FEL and laser acceleration experiments at ATF. With program PARMELA 5] With $\sigma_{r}=1 \mathrm{~mm}$ and $0.9 \mathrm{~mm}$ (uniform beam distribution) we obtained beam emittance of few tenths of cm-mrad with energies of about $46 \mathrm{MeV}$ and brightness $\left(B=I^{\text {peak }} / \pi \epsilon_{x}^{V} \epsilon_{y}^{V}\right)$ of orders of $10^{13}$ for the beam emerging from the exit of the linac, (Fig.4). We have used $10 \mathrm{ps}$ pulse length in our analysis since $\sigma==5$ ps is the value noted obtainable at ATF [1].

For $B_{0}=2.14 \mathrm{KG}, \sigma_{z}=5 \mathrm{ps}, \sigma_{r}=1 \mathrm{~mm}, Q=1 \mathrm{nC}$ and an initial uniform distribution, the beam energy and emittance at the linac exit become $w=46 \mathrm{MeV}$, $\epsilon_{x}^{v, r m s}=.32$ and $\epsilon_{y}^{V, r m a}=.276$. Whereas for the same set of parameters and an initial gaussian distribution the beam emittance grows larger, e.g. at the linac exit. How easy is the production of the uniform distribution (flat beam) depends on the quality and bandwidth of the laser [1]. For detailed beam analysis with Gaussian and Rician distributions see e.g. $[1,6]$.

Fig. 4 shows the change in the beam size along the beamline from cathode through the linac. As can be seen the beam converges to a waist in the linac. Where the change in the solenoid strength or $d$ would shift the position of the beam waist in the linac and would change the beam parameters. For example a $2 \%$ increase in the solenoid strength will increase the emittance from $\left(\epsilon_{x}^{V . r m s}=.278, \epsilon_{y}^{V, r m s}=.243\right)$ to $\left(\epsilon_{x}^{V, r m s}=390\right.$. $\left.\epsilon_{y}^{\mathrm{V}, r m s}=333\right)$ at the linac exit. keeping all other parameters fixed [1].

For the proposed alternate injection system for ATF, a pair of solenoids are placed before and after the gun to compensate the beam emittance dilution due to the space charge, such that the coil proceeding the cathode bucks the field due to the second solenoid and produces a zero field on the cathode. To avoid beam loss through the linac a proper matching of the beam into the linac is crucial. 


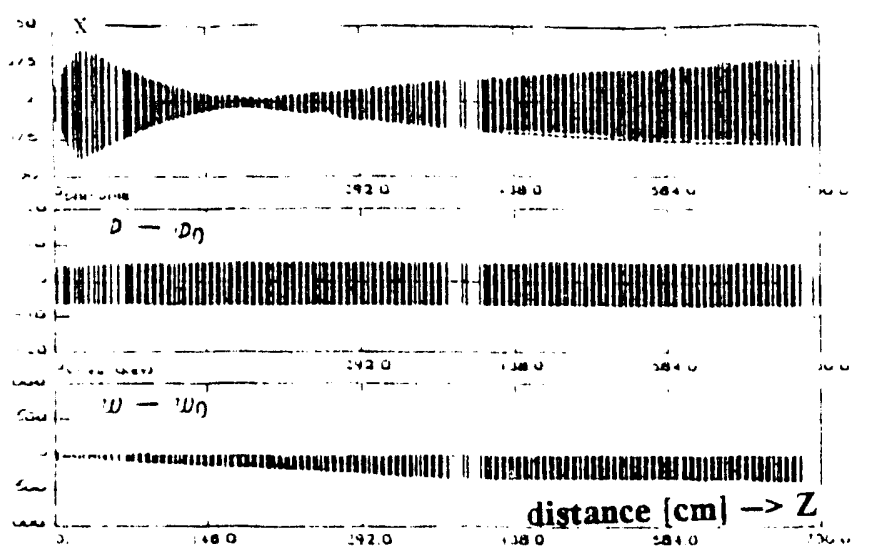

Figure 4: Shows the change in position $x[\mathrm{~cm}$, phase $\Phi-\Phi_{0}$ idegreej and energy $w-w s[\mathrm{KeV}]$ of particles at each element location, from the cathode through the linac exit. With $2.2 \mathrm{KG}$ solenoid, $d=70 \mathrm{~cm}, \sigma_{\tau}=.9 \mathrm{~mm}$ and $\sigma_{z}=$ sps.

\section{SUMMARY}

We presented an alternate injection system for the ATF at Brookhaven National Lab, using a pair of solenoids and an if gun placed directly into the linac. Earlier results showed that with the present injection system at ATF the beam become too large through the double bend transport line, leading to a large emittance growth and a large beam loss. With solenoid+gun+solenoid straight injection into the linac scheme we can reduce the emittance dilution due to space charge forces, and produce the beam needed for EEL, IFEL and other laser acceleration experiments. We obtained small emittance (few tenths of $\mathrm{cm}$-mrad) and high brightness of orders of $10^{13}$. We note that a proper beam matching at the linac entrance is crucial so as to avoid beam loss through the linac. The effects of variations in the solenoid strengths and $d$ the distance from the cathode to the linac entrance can be detrimental to the beam quality along the transport line and through the linac. The beam converges into a waist after it enters into the linac. The position of this waist changes with the change in initial parameters such as the solenoid strength and the distance between the cathode and the linac entrance. To avoid beam loss or emittance dilution, there must be a careful control of the parameters such as the length and strength of the solenoids, laser spot size and puise length, distance between the cathode and linac entrance, the accelerator phase and accelerator field. In this analysis the fields off axis for solenoids were calculated to $6 \mathrm{th}$ order.

\section{REFERENCES}

-Work performed under the auspices of U.S. Dept.of Energy contract DE-AC02-76-CH00016.

i. Z. Parsa, "Preliminary Emittance Measurements and Calculations of the Laser Excited Beam from the BNL Accelerator I'est Facility Radio Frequency Electron Gun", Vol.II, Sec.II, p1-j4, BNL-52292 (1991) and References therein; K. McDonald et al., Proc.,
Table 1: ATF Solenoid+Gun-Solenoid-Linac system simulation parameters.

\begin{tabular}{|c|c|}
\hline Number of Solenoids & 2 \\
\hline Length of Solenoid $\mathrm{cm}$ ! & 15 \\
\hline Radius of Solenoid $i \mathrm{~cm}$ ! & $\hat{j}$ \\
\hline$B_{n}$ Solenoid $[K G !$ & 2.2 \\
\hline Initial phase idegree! & $43-45$ \\
\hline Laser spot size $\sigma_{r} \mathrm{~mm} !$ & $.9,1 \ldots$ \\
\hline Laser Pulse Length $\left(2 \sigma_{z}\right)$ psi & 10 \\
\hline RF Frequency $[\mathrm{GHz}]$ & 2.856 \\
\hline Radius of Aperture $\mathrm{cm}$ ! & 1.0 \\
\hline Field on the Cathode $[\mathrm{MV} / \mathrm{m}]$ & 100 \\
\hline Charge $[\mathrm{nC}]$ & 1 \\
\hline with $\mathrm{d}[\mathrm{cm}]$ & $62-70$ \\
\hline Linac Accel. Gradient (Ave.) $[\mathrm{MV} / \mathrm{m}]$ & 7.0 \\
\hline At Linac Exit: Energy $[\mathrm{MeV}]$ & $46-47$ \\
\hline $\mathrm{dp} / \mathrm{p}[\%]$ & $0.18-0.2$ \\
\hline$\epsilon^{v}[\pi \mathrm{cm}-\mathrm{mrad}]$ & $0.2-10.4$ \\
\hline Brightness $\left(B=I^{\text {peak }} / \pi \epsilon_{x}^{\vee \vee} \epsilon_{y}^{\vee}\right)$ & $\times 10^{13}$ \\
\hline
\end{tabular}

EPAC88, June 1988; A. Fisher, J. Fisher, I. pogorelsky, private communications on laser performance.; Z. Parsa, "Effects of 3rd Order Maps and Solenoid on a Bigh Brightness Beam", BNL-48397 (1993) and Proc. IEEE PAC May 17-20, Washington D.C. (1993).

2. K. Batchelor, I. Ben-Zvi, I. Bigio, T.S. Chou, R.C. Fernow, J. Fischer, J. Gallardo, E. Kirk, N. Kurnit, K.T. McDonald, R.B. Palmer, Z. Parsa, C. Pellegrini. J. Sheehan, T. Srinivasan-Rao, S. Ulc, A. Van Steenbergen, M. Woodle, "Operational Status of Brookhaven Accelerator Test Facility “, Proc. March 20-23,1989 IEEE PAC, Chicago, Ill, \#89CH2669-0 (1990).

3. Z. Parsa, "On Beam Emittance and Invariants - Applications to ATF Beamline", Proc.2nd EPAC, NiceFrance, Nile Ed., Frontiers, (1990); BNL Report BNL44364 (1990) and Ref.therein.

4. Z. Parsa, and L. Young, "Modeling of the BNL Photocathode Gun with the Code IARMELA “. Vucl. Inst. \& Meths. A318 (1992) 254-258.

5. L. Young, (Private comms.), PARMELA, 1993 version; We thank L. Young for helpful discussions. modifications to the programs and collaborations.

6. Z. Parsa, "An Alternate Injection System for the BNL Accelerator Test Facility", BNL-48403, APS April 1215, Washington D.C. (1993); Z. Parsa, "Interactzons Between Charged Particles and RF Space Charge and Wake Fields in an Accelerating Structure". Particles and Fields 91, Vol. 2, 1073, Ed. David Axen, Douglas Bayman and Martin Comyn; Z. Parsa, Accelerator Physics and Modeling, BNL- Report BNL-52379. 55165 (1993). 

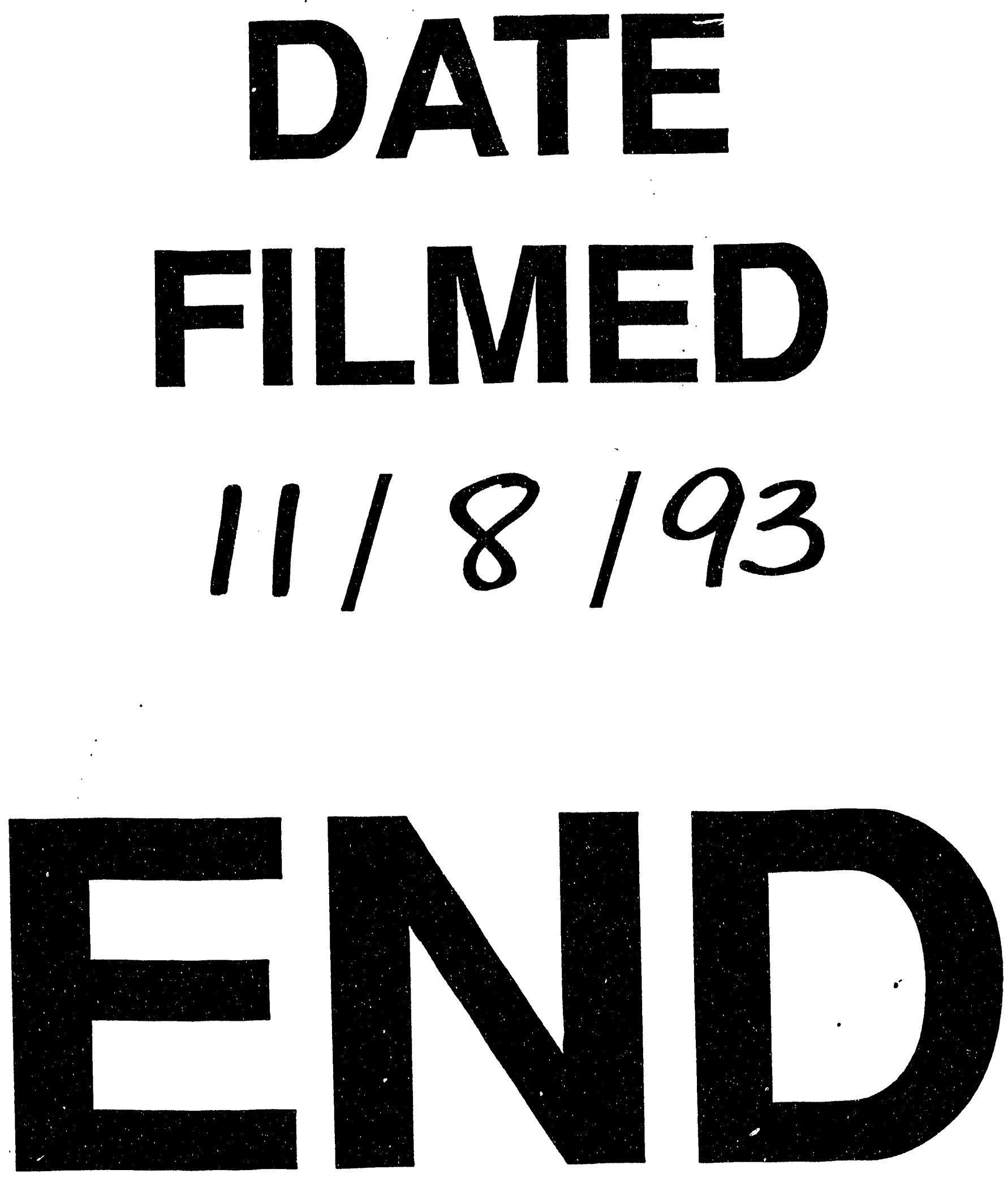
